Franco López, J. A. (septiembre-diciembre, 2021). La motivación docente para obtener calidad educativa en instituciones de educación superior. Revista Virtual Universidad Católica del

Norte, (64), 151-179. https://www.doi.org/10.35575/rvucn.n64a7

\title{
La motivación docente para obtener calidad educativa en instituciones de educación superior
}

The teaching motivation to obtain educational quality in higher education institutions

\section{Jorge Ariel Franco López}

Magister Administración (MBA)

Facultad de Ciencias Económicas y Administrativas, Instituto Tecnológico Metropolitano -ITMMedellín, Colombia

jorgefranco@itm.edu.co

Orcid: http://orcid.org/0000-0002-0507-2914

CvLac: https://scienti.minciencias.gov.co/cvlac/visualizador/generarCurriculoCv.do?cod rh=0001457457

Recibido: 12 de octubre de 2020

Evaluado: 19 de noviembre 2020

Aprobado: 13 de abril de 2021

Tipo de artículo: Investigación Científica y Tecnológica

\section{Resumen}

El profesional de la educación tiene un gran compromiso social: la formación de sus educandos para enfrentar el proyecto de vida; desde esa perspectiva, el docente de instituciones de educación superior (IES) debe mantener un alto grado de motivación, entendiéndose como esa fuerza que hace moverse y surge por necesidad de lograr mejores estándares; esencial en los instructores de educación superior, para tener alta calidad en el proceso formativo y participar adecuadamente en el futuro profesional. El objetivo desarrollado fue analizar qué categorías o variables motivan a los docentes de IES para obtener calidad en la educación. La pesquisa bibliográfica permitió construir un sistema de catorce categorías. Fue una investigación descriptiva con método cualitativo, donde se utilizó una metodología con la técnica Delphi, donde diez expertos con formación en docencia universitaria, quienes analizaron a través de dos rondas un sistema categorial, concluyeron que las 


\title{
La motivación
}

docente

categorías a evaluar fueran: factores extrínsecos e intrínsecos, transformar conocimiento, calidad en la educación, formación permanente, y docencia como generadora de estatus social. Todas fueron analizadas a través de una hermenéutica para cada categoría, llegando a concluir que son los factores extrínsecos, seguidos de los intrínsecos, aquellos los más decisivos en la motivación de los docentes en IES.

Palabras claves: Calidad en la educación; Docente universitario; Estrategia educativa; Instituciones de educación; Motivación.

\begin{abstract}
The education professional has a great social commitment, training their students to face the life project; From this perspective, the teacher of higher education institutions (IES) must maintain a high degree of motivation, understood as that force that makes them move and arises from the need to achieve better standards, essential in higher education instructors to have high quality in the training process and participate adequately in the professional future. The objective developed is to analyze which categories or variables motivate IES teachers to obtain quality in education. The bibliographic search allowed to build a system of fourteen categories. It is a descriptive research with a qualitative method, using a methodology with the Delphi technique, where ten experts with training in university teaching, analyzed a categorical system through two rounds, they concluded that the categories to be evaluated were: extrinsic and intrinsic factors, transform knowledge, quality in education, permanent training, and teaching as a generator of social status. All were analyzed through a hermeneutic for each category, reaching the conclusion that extrinsic factors, followed by intrinsic factors, were the most decisive in motivating teachers in IES.
\end{abstract}

Keywords: Quality in education; University teacher; Educational strategy; Educational institutions; Motivation 


\section{La motivación}

docente

\section{Introducción}

La educación representa para todas las sociedades uno de los aspectos más relevantes para obtener niveles de crecimiento, desarrollo social y económico, "cuando se observan las razones centrales que han permitido a los países no quedarse estancados y adheridos sólo al crecimiento, sino que también vislumbran, logran y disfrutan del desarrollo; aparece un elemento central, el factor educación" (Ganga et al., 2018); es decir, la educación es una fuente vital para obtener bienestar, especialmente para aquellas sociedades que logran tener procesos formativos rigurosos (Arias-Ciro, 2020). Ahora, para el caso de las instituciones de educación superior (IES) presenta varios aspectos coyunturales que deben resolverse; sin embargo, se destacan dos aspectos: cobertura y calidad.

De la cobertura, es tratar de llegar a la mayor cantidad de personas de la población; de hecho, la educación debe ser un compromiso, no solo de interés para el Gobierno, a través de la propuesta pública, sino, además, para el mercado, con la gestión privada. El otro aspecto para detallar es la calidad en la educación, que debe ser un compromiso de todos los actores involucrados en el proceso; sin embargo, "los docentes son los grandes pilares de la educación de calidad" (R. Martínez et al., 2020). Y es precisamente en esta última parte donde se quiere ahondar en la propuesta de investigación. Actualmente las IES buscan obtener reconocimiento gubernamental y de la comunidad académica, a través de procesos de acreditación, que la destaquen por tener calidad en los programas y en el desarrollo organizacional. Como lo indican Orozco et al. (2020) (categoría 1), existen dos formas para lograr calidad por parte de las IES: i) a través del aseguramiento externo, que proviene de organismos de control, como el caso de autoridades gubernamentales educativas; y ii) la adopción de políticas internas que aseguren una cultura de la calidad; es decir, todos sus actores involucrados en el proceso de calidad.

Sobre el tema de la calidad hay múltiples definiciones, una de ellas "es cuando satisface las necesidades y expectativas del cliente o usuario, en función de parámetros como: seguridad, fiabilidad y servicios" (Pola, 1988); para el caso de la calidad en la educación, "puede ser entendida como el valor que se le atribuye a un proceso educativo según parámetros establecidos por la sociedad o entidades rectoras en educación, que permiten una calificación como mejor, igual o menor que las de sus pares" (Asencio et al., 2019) (categoría 2). El Ministerio de Educación 
Nacional colombiano (2016), establece en el Compendio Estadístico de la Educación Superior colombiana, que el Estado adopta y hace público el reconocimiento mediante la revisión de pares académicos, y la comprobación de calidad otorgada a las IES. Para ese cumplimiento efectúa una serie de doce (12) factores, y cada uno de ellos con las debidas características, los cuales deben reunirse para otorgar la acreditación de programas y de las instituciones. En ese sentido, el Acuerdo 02 de 2020, sobre la acreditación de alta calidad que otorga el Ministerio de Educación Nacional, actualiza el modelo de acreditación, a fin de que toda la comunidad académica cumpla con condiciones óptimas: estudiantes, profesores, egresados, empleados, directivos y grupos sociales que tengan relación indirecta con la institución. "Transformar el sistema educativo, permitiendo el acceso a la educación y ofertando una formación de calidad, es uno de los ideales más importantes presentes en la última década en los países latinoamericanos" (Loaiza-Aguirre y Andrade-Abarca, 2021).

El Acuerdo 02 de 2020, establece en el tercer (3) factor que los docentes son pieza vital en la acreditación; allí se establecen las condiciones como el nivel académico, la dedicación, el tipo de vinculación, la calidad y pertinencia; igualmente, hace mención del desarrollo académico y pedagógico, a la permanencia y cualificación docente, en términos de formación y producción. El factor establece ocho (8) características: selección, vinculación y permanencia; estatuto profesoral; número de docentes, tipo dedicación, niveles de formación y experiencia; desarrollo profesoral; estímulos a la trayectoria; producción, pertinencia e impacto del material; remuneración por méritos; y evaluación de profesores.

Desde esa perspectiva, se quiere establecer al docente como uno de los factores de calidad en el proceso de capacitación y formación; no el único, obviamente, pero sí condición básica que determina la ruta de aprendizaje a seguir por parte de los dicentes, "un profesor realiza día tras día, la defensa de la veracidad, y la objetividad a la hora de explicar las ideas, mostrar respecto ante la diversidad cultural y social, el compromiso con su formación permanente" (Bara, 2018) (categoría 3). El docente juega un papel importante en los procesos formativos, no solo por ser transmisor de conocimiento, sino porque alberga el carácter sensible de la ciencia y la estimulación que debe tener una persona desde la estructura de valores, "Las relaciones basadas en el cuidado, la confianza, el respeto, el afecto, la apertura y la cooperación pueden fomentar el logro estudiantil, 


\section{La motivación}

docente

el desarrollo de la identidad y la pertenencia a la escuela" (A. Ibrahim \& Zaatari, 2020) (categoría 4).

En este orden de ideas, el objetivo de la investigación es demostrar qué categorías o variables motivan a los docentes de IES para obtener calidad en la educación. Ahora, hablar de calidad es difícil, por las múltiples variables que la rodean; sin embargo, se ha querido precisar una de las que se ha venido trabajando por parte de la comunidad académica. La figura 1 presenta algunos datos estadísticos, con respecto a la formación de los docentes en Colombia:

\section{Figura 1}

Niveles de formación académica de la población docente en Colombia 2018-2

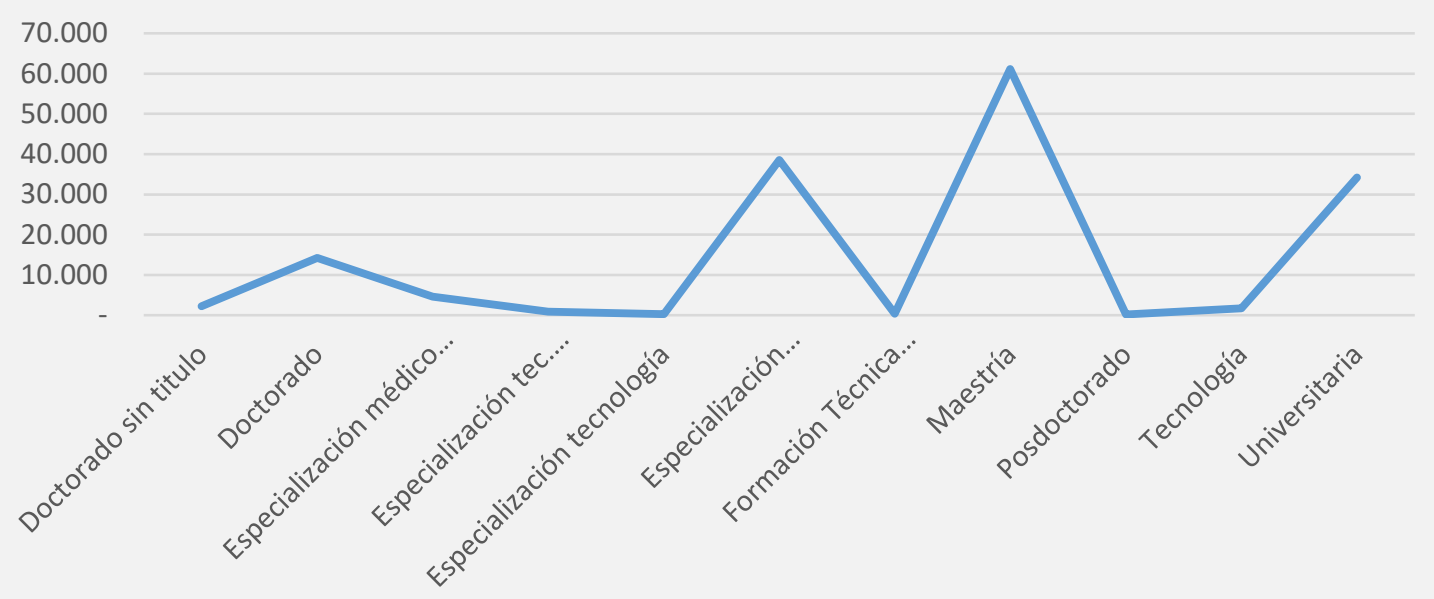

Nota: estadística SNIES (2020); adaptación propia.

La formación de los docentes en Colombia en las IES se concentra, para 2018-2, en la formación de maestría, con un 38,57 \%; y en especialización universitaria, con un 24,33 \%. La formación de los docentes es un factor básico (no suficiente) para obtener calidad en la formación en los estudiantes.

De otro lado, la actividad docente, dice Alexander (2008) (categoría 5), es desafiante; además, esos desafíos son internos y externos, los retos son muchos y exigentes, porque, sobre el docente recae, en su gran mayoría, la calidad en el proceso. La responsabilidad que tiene el docente es de gran exigencia; el compromiso adoptado es sobre un contexto de personas que han puesto la 


\section{La motivación}

docente

confianza sobre él y la institución; por eso, es vital mantener un alto nivel de motivación para efectuar bien la tarea, "motivación ha sido generalmente considerada como la energía o la unidad que mueve a la gente a hacer algo por la naturaleza" (Han \& Yin, 2016). Desde otra perspectiva, Thoonen et al. (2011) desarrollan un modelo de relaciones entre los factores de la motivación, que influyen indirectamente en la calidad de todas las actividades del aprendizaje.

Otro aspecto que liga la investigación es la pregunta: ¿qué tipo de motivación requiere un docente en una IES para obtener calidad en el proceso formativo? Pues bien, los cambios históricos dentro de los distintos ciclos han indicado que los movimientos se deben básicamente a los cambios tecnológicos, producto de la innovación. Describe Penprase (2018) (categoría 6) que, a diferencia de otros sectores productivos, el impacto que genera el cambio tecnológico en la educación requiere más tiempo para la adaptación; sin embargo, esta cuarta revolución es diferente a las anteriores, en Estados como la India y Estados Unidos indica que la población con algún acceso a la educación superior ha aumentado del 4\% en 1900 a casi el 70\% en 2000.

En ese sentido, el docente tendrá, en lo inmediato, disposición a nuevas tendencias en el aprendizaje; mostrar otro grado de motivación, como la inteligencia artificial, el aprendizaje autónomo, robótica, nanotecnología, genética, teletrabajo, biotecnología, impresión 3D, genómica, información al instante, proliferación de las redes, nuevas pedagogías, aprendizaje en línea, equipos de alto rendimiento, aspectos ambientales, la nube, big data, entre otros. Todo esto tiene un impacto no solo en lo económico, sino en todas las instancias de la sociedad y del saber; "Industria 4.0 es el intento de hablar ideológicamente de un nuevo paradigma tecnológico" (Fuchs, 2018).

Bajo la argumentación anterior, es claro que el trabajo centra la atención en la educación como actividad fundamental, y permite el constructo de mejores condiciones de vida de las sociedades; y dentro de ese sistema, en el cual participan varios agentes, la atención estará colocada en la motivación de los docentes en las IES, como organizadores y orientadores que gestan los procesos de formación y capacitación, en términos de calidad educativa. Dentro de la actividad, Pan \& Hu (2020) destacan que los docentes mantienen una enorme presión, con lo cual existe un agotamiento laboral, y mencionan el capital psicológico (CP), temática que también es trabajada por Gong et al. (2019), cuando indican que el CP tiene un efecto predictivo negativo sobre el agotamiento del docente (en este caso), y un efecto predictivo positivo sobre el desempeño laboral; 


\section{La motivación}

docente

además, la inteligencia emocional ayudará a los docentes a mejorar el $\mathrm{CP}$ de la institución. La literatura también reconoce el agotamiento laboral como el síndrome de burnout, o también del profesor quemado; lo anterior, según González-Torres (2003, como se citó en Franco, Vélez et al., 2018), se refiere a las afecciones psicosociales de la persona al exponerse a períodos de estrés prolongados. Todo lo anterior, arroja la interrogante: ¿Cuáles son las categorías o variables que motivan a los docentes de la IES para generar procesos de calidad educativa?

\section{Estado del arte}

Han \& Yin (2016), (categorías 7 y 8) determinaron cinco áreas de investigación en el tema de la motivación docente: 1) los factores que influyen, 2) la motivación docente como factor de eficacia en la enseñanza, 3) la motivación de los estudiantes en el proceso formativo, 4) la motivación docente en las diferentes disciplinas, y 5) la creación de instrumentos evaluativos. En la amplia revisión de la literatura, por parte de los autores, destacan cuatro aspectos trabajados en las distintas investigaciones sobre el tema de la motivación de los docentes: 1) Motivación intrínseca, 2) influencia del entorno social y sus limitaciones, 3) La importancia del proceso de formación en el proyecto de vida de cada persona, y 4) los factores negativos que generan desmotivación. La tabla 1 representa una síntesis sobre la posición teórica de varios autores sobre el tema de la motivación, desde la perspectiva de Han \& Yin (2016).

\section{Tabla 1}

Visiones de autores sobre la motivación de los docentes

Autor

(Sinclair, 2008)

Dörnyei y Ushioda, 2012)

\section{Descripción}

Es la motivación lo que atrae a ciertas personas a las actividades docentes, y es la relación que se establece entre los cursos y la profesión de la enseñanza. Categorías de la motivación: llamadas, estudiantes, altruismos, simulación intelectual, influencia en los demás, conveniencia de la enseñanza, naturaleza del trabajo docente, cambio, facilidad para enseñar, estado de la enseñanza.

La motivación para enseñar y permanecer en la profesión. Destacan 4 componentes citados inicialmente. Indican cinco factores de desmotivación: estrés, inhibición a la autonomía y estructura de carrera, insuficiencia de la autoeficacia, contenidos repetitivos, y limitado desarrollo intelectual. 
(Brookhart y

Freeman, 1992)

(categoría 5)
El estudio representa la motivación de los docentes que inician sus actividades y lo ligan en relación con cuatro variables: aspectos demográficos y antecedentes de la escuela a desarrollar la actividad; expectativas; confianza y optimismo sobre la educación; y responsabilidad en la enseñanza.

Teoría de las expectativas de valor FIT- Choice (Factores que influyen en la elección de la enseñanza). Estudio hecho en las Universidades Australianas. Recomiendan estratégicamente el reclutamiento de personas para enseñar, dependiendo del grado motivacional. El modelo incluye que la elección de la profesión es el resultado final de auto percepciones, valores, estatus social, salario, aspectos intrínsecos como utilidad personal y social.

(Richardson \&

Watt, 2006)

(categoría 8)

La motivación de una persona para volverse maestro es el deseo de impartir conocimiento al servicio de la sociedad.

La motivación de los docentes en su servicio radica en mejorar la motivación de los estudiantes, manejar las reformas educativas y cumplir con los otros docentes. El trabajo se ubica dentro de las teorías cognitiva - motivacionales, efectuado en escuelas de Portugal.

(De Jesus \& De Willy, 2004)

(Praver \& OgaFactores intrínsecos para maestros en formación, extrínsecos para maestros en

Baldwin, 2008) (categoría 9) servicio (salarios, pensiones, seguros) e indirectos, direccionan la motivación docente.

(Atkinson, 2000) Los docentes (Turquía) tienen varios aspectos de desmotivación: administración educativa, estudiantes, bajos salarios, pocas oportunidades para investigar.

Nota: adaptación propia de Han \& Yin (2016).

De otro lado, el trabajo de Kiziltepe (2008) describe que la motivación es un tema trabajado desde la psicología y la educación. Es la motivación esa fuerza que hace moverse, surge por la necesidad para lograr mejores estándares; es un comportamiento humano que se compone de fuerzas internas reflejadas en energía psíquica. Allí, el enfoque inicialmente se efectúa en la motivación en el trabajo de distintos autores, tales como: Taylor, Mayo, Maslow, Herzberg, Mausner y Snyderman, Herzberg, Evans, Leung Si y Spector, quienes hicieron contribuciones frente al concepto motivación fundamental en las explicaciones del entramado organizacional. Por su parte otros trabajos puntualizaron la motivación en los docentes como Holdaway, Person \& Seiler, La Colina, Lacy y Sheehan, y Calderhead y Shorrock. Aspectos que generan desmotivación en los docentes se encuentran en los trabajos de Oshagbemi, Ssesanga y Garrett, Gruneber. Dentro de la motivación intrínseca se destaca la vocación, en autores como Hansen, Hill, Lomas y MacGregor, Staniforth y Harland, Estola, Erkkila y Syrjala.

La investigación de M. Ibrahim \& Nat (2019) (categorías 8, 9 y 10) destaca la tendencia en los procesos de formación y capacitación, con el uso de las tecnologías de la información y comunicación (TIC); más concretamente con Blended Learninig, tendencia utilizada en las 


\section{La motivación}

docente

instituciones de educación superior (IES) que impacta positivamente en el rendimiento para los estudiantes. Sin embargo, el éxito dependerá del grado de motivación que tengan los docentes en incursionar en ellas; además, el tener el apoyo de la institución en la adquisición de la tecnología a utilizar y de un programa de capacitación a los docentes (alfabetización tecnológica). De acuerdo a la motivación, los autores destacan, en la motivación extrínseca, la interacción de los docentes con la tecnología, el número de horas de clase, los estudiantes, el compromiso institucional; y factores intrínsecos, como aptitudes, creencias, aprendizaje.

Destacan Fong et al. (2019) (categoría 10), que la motivación es esencial en los instructores de educación superior para tener alta calidad en los estudiantes y participar en el futuro profesional. Por su parte, E. Rodríguez et al. (2013) (categoría 9) muestran dos tipos de motivación: controlada, la cual se asocia con conductas para evitar consecuencias negativas, en aras de obtener recompensas; y la motivación autónoma, que es una experiencia interna, propia de la identidad, donde hay una identificación axiológica, que asocia también la persistencia que mejora los procesos. La motivación es un estado que estimula el deseo por actuar o dirigir la atención de los estudiantes; es decir, los docentes tienen el papel de motivar a los aprendices en la adquisición del conocimiento, donde hay un aprendizaje cognitivo que tiene que ver con la capacidad de comprender y aplicar el conocimiento, pero también existe un aprendizaje afectivo que tiene que ver con las actitudes y sentimientos de los estudiantes. Lo cognitivo se logra por intermedio de lo afectivo.

Por su parte, Hotaman \& Yüksel (2010) (categoría 11), indican el rol que desempeñan los docentes en la vida de sus estudiantes; es vital mantener el entusiasmo para captar la atención; "Ciertos educadores consideran el entusiasmo como un rasgo de la personalidad innata, mientras que otros tienden a considerarlo como una habilidad adquirida. Sin embargo, ambas perspectivas están de acuerdo en el punto sobre el entusiasmo" (p. 90). Además, Cohen \& Lawrance (1989, como se citó en Hotaman \& Yüksel, 2010), indicaron que existen también otros aspectos motivantes de los docentes a los estudiantes, tales como la voz, comportamiento y expectativas, sistema de creencias, sentido del humor, liderazgo, técnicas de control, y habilidades de apreciación. De igual manera, Burden \& Byrd (1994, como se citó en Hotaman \& Yüksel, 2010) afirman que el docente, para mantener el entusiasmo, debe tener ciertas pautas frente a sus 
estudiantes: presentación verbal, contacto visual, gestos, vocabulario, probación de ideas y sentimientos, nivel de energía.

De otro lado, la investigación de Bailey (1999) (categorías 12 y 14) destaca la motivación y desmotivación en los docentes del inglés como lengua extranjera, identificando factores motivantes en la práctica docente, tales como: estudiantes, programa académico, estatus social e ideales; por su parte, factores desmotivadores como: aspectos financieros, características físicas de la institución, condiciones para investigar y desarrollar las otras actividades docentes. El trabajo de Sugino (2010) menciona la desmotivación que sufren 97 docentes de Japón, demostrando que las principales causas obedecen a las aptitudes y competencias de los estudiantes, al igual que el plan de estudios; "Los maestros también se sienten motivados cuando ven la motivación y el progreso de los estudiantes y cuándo son apreciados por éstos” (p. 216). La investigación de Baleghizadeh \& Gordani (2012) demostró la relación entre la calidad laboral y la motivación de los docentes de inglés como lengua extranjera, en Irán; habla el estudio de la importancia que tienen las autoridades gubernamentales y la administración de las instituciones educativas en ofrecer calidad de vida a los docentes, de tal manera que generen motivación para tener actividades de calidad en la enseñanza. En la propuesta de Tae et al. (2014), se muestran los diferentes aspectos de desmotivación entre profesores de inglés, chino y coreano; entre ellos están, las muchas actividades laborales y la falta de formación profesional en los docentes de la lengua inglesa; sin embargo, en los sistemas educativos de ambos países, la formación en este idioma es una exigencia. Se destaca el trabajo que hacen en dos países, como China y Corea, los cuales comparten ciertos aspectos culturales, como la herencia confucionista y un alto valor a la educación.

En Fattash (2013) se destacan tres teorías que explican la motivación docente: a) teoría de las expectativas, en la cual el empleado labora de acuerdo a una recompensa esperada, b) teoría de la equidad, en la que el no reconocimiento en los esfuerzos genera desmotivación., y c) teoría del enriquecimiento laboral, cuanto variada y exigente sea la actividad laboral, los docentes serán más productivos. Destacan Deci \& Ryan (2008) sobre otra teoría de la autodeterminación, la cual centra la atención en la motivación autónoma, controlada, y predictores de rendimiento; la teoría cuestiona aspectos básicos del desarrollo de la personalidad, la autorregulación, las necesidades psicológicas, procesos no conscientes, relaciones culturales, procesos sociales, efecto y bienestar. La teoría se ha aplicado en distintos estudios. 


\section{La motivación}

docente

Por otro lado, en la investigación de C. Martínez et al. (2017) (categoría 13), se indica que el docente, como agente transformador del conocimiento, debe contar con una serie de capacidades y habilidades para llevar a cabo sus actividades laborales; una de ellas es la motivación, "apreciar que esta encrucijada en la que se encuentra el docente, no es de naturaleza lógica, sino que se asocia a diversos componentes (afectivos, experiencias, ámbito social...); también en base a la diversidad de contextos (psicológicos, sociales, culturales...)” (p. 77). También, el estudio de Horodnic \& Zait (2015) (categorías 8, 9 y 13) correlaciona la productividad en investigación con respecto de la motivación intrínseca y extrínseca, debido a la importancia que tiene la investigación en la visibilización, especialmente en las IES; se llega a considerar "que los investigadores que están fuertemente motivados por las recompensas (tanto intrínsecas como extrínsecas) publican significativamente más artículos en revistas" (p. 285). Complementando el tema de la productividad en las IES, el trabajo de Nie et al. (2012) indica que la autoeficacia del docente es la capacidad para lograr compromisos deseados y del aprendizaje del estudiante, todo esto a través de tres factores: la eficacia para la instrucción, manejo del aula, y especialmente la eficacia en la motivación.

En otra fuente, López et al. (2017) presentan una investigación exploratoria hecha a personal docente administrativo de colegios, sobre la motivación de docentes en cuatro áreas específicas: dotación institucional, aspectos intrínsecos y extrínsecos e impactos sociales. Se concluye que el factor más decisivo en la motivación de los docentes, indicada por los directivos institucionales, es lo intrínseco, concretamente la vocación. De igual manera, Franco, López et al. (2020) (categoría 8), en un estudio de tipo correlacional, indican que hay una correlación positiva entre la motivación de los docentes, con respecto de los salarios, aspectos intrínsecos y reconocimiento social. Doyle \& Kim (como se citó en Bailey, 1999) (categoría 14) señalan en su estudio que factores como el salario, la relación con los administrativos docentes, el avance en los planes de estudio, la falta de libertad de cátedra, y la estabilidad en el empleo, son factores que, según como se presenten, generan motivación o desmotivación en los docentes. La investigación concluyó que existen factores que generan desmotivación en los docentes: problemas con el plan de estudio, relaciones con los estudiantes, administrativos y otros docentes, las condiciones contractuales de trabajo, condiciones físicas. Dorney (2001, como se citó en Fattash, 2013) (categoría 9), presenta cuatro aspectos motivacionales de los docentes: componente intrínseco, 


\section{La motivación}

docente

factores contextuales, dimensión temporal e influencias negativas. De la motivación intrínseca, es realización y crecimiento personal, es decir, el grado de seguridad emocional.

\section{Metodología}

La investigación llevada a cabo determinó un método de tipo cualitativo, donde "se concibe lo social como una realidad construida que se genera a través de articulaciones con distintas dimensiones sociales, es decir, por una diversidad cultural sistematizada" (Guerrero y Guerrero, 2014); en ese sentido, la educación es una relación que se establece en todo el tejido social, y en todas las organizaciones que lo componen. Desde esa perspectiva, para el objeto de estudio de la investigación, los docentes son actores importantes en el proceso de enseñanza - aprendizaje, en los entornos organizacionales como son las IES. Siendo la motivación un aspecto comportamental que determina el actuar de todos los docentes en sus distintos ámbitos de desempeño institucional, amerita para su estudio un análisis que confronte las distintas propuestas teóricas, descritas en el estado del arte, y los hallazgos a desarrollar en el trabajo de campo.

Para llevar a cabo la investigación, desde el punto de vista metodológico, se abordó el estado del arte y la propuesta de Galeano (2004), la cual es una ruta para trabajos cualitativos que consiste en sacar un sistema categorial, lo suficientemente representativo, que valide la categoría o variable, como es la motivación de los docentes en IES, con el ánimo de obtener calidad en el proceso formativo. La tabla 2 precisa el sistema categorial extraído desde el soporte bibliográfico, abordado en parte inicial.

\section{Tabla 2}

Sistema categorial seleccionado inicialmente

\begin{tabular}{|c|c|c|}
\hline No. & Categorías & Soporte bibliográfico \\
\hline 1 & $\begin{array}{l}\text { Acreditación de programa } \\
\text { institución }\end{array}$ & e (Orozco et al,, 2020) \\
\hline 2 & Calidad en la educación & (Asencio et al., 2019) \\
\hline 3 & Formación permanente & (Bara, 2018). \\
\hline 4 & Cuidado, confianza & (A. Ibrahim \& Zaatari, 2020). \\
\hline 5 & Responsabilidad en la enseñanza & (Alexander, 2008) (Brookhart y Freeman, 1992 \\
\hline 6 & Cambios tecnológicos & (Penprase, 2018) \\
\hline
\end{tabular}




\section{La motivación}

docente

\begin{tabular}{lll}
\hline $\mathbf{7}$ & Eficacia en la enseñanza & (Han \& Yin, 2016) \\
\hline $\mathbf{8}$ & Factores intrínsecos (vocación) & $\begin{array}{l}\text { (Richardson \& Watt, 2006) } \\
\text { Ibrahim \& Nat, 2019) (Horodnic \& Zait, 2015) } \\
\text { (Franco, López et al., 2020) }\end{array}$ \\
\hline $\mathbf{9}$ & $\begin{array}{l}\text { Factores extrínsecos (salario, } \\
\text { pensiones) }\end{array}$ & $\begin{array}{l}\text { (Praver \& Oga-Baldwin, 2008) (M. Ibrahim \& Nat, } \\
\text { 2019) (E. Rodríguez et al., 2013) (Fattash, 2013) } \\
\text { (Horodnic \& Zait, 2015) }\end{array}$ \\
\hline $\mathbf{1 0}$ & Rendimiento de los estudiantes & (M. Ibrahim \& Nat, 2019) (Fong et al., 2019) \\
\hline $\mathbf{1 1}$ & $\begin{array}{l}\text { Entusiasmo para captar la atención } \\
\text { (Hotaman \& Yüksel, 2010) }\end{array}$ \\
\hline $\mathbf{1 2}$ & $\begin{array}{l}\text { La docencia como generadora de } \\
\text { status social }\end{array}$ & $\begin{array}{l}\text { Transformar conocimiento, generar } \\
\text { (Bnvestigación }\end{array}$ \\
\hline $\mathbf{1 3}$ & Libertad de cátedra & (C. Martínez et al., 2017) (Horodnic \& Zait, 2015) \\
\hline $\mathbf{1 4}$ & (Bailey, 1999)
\end{tabular}

Nota: elaboración propia.

Del sistema categorial seleccionado, como referente para el análisis de la motivación de los docentes de IES, en su parte explicativa, para cada una de las categorías, se tiene:

1) Acreditación de programas e instituciones como estrategia de mejoramiento de la calidad por parte del Gobierno. No es obligatoriedad por parte de las IES tener estos reconocimientos; sin embargo, no tenerlos es estar por fuera del mercado educativo, en el ámbito nacional e internacional.

2) De la calidad educativa, son múltiples los aspectos transversales que forman parte del proceso de formación; cabe mencionar algunos que contribuyen a tener estándares de calidad: se puede corroborar con los egresados, como indica Serrate et al. (2019) la interrelación entre la formación teórica y el mundo laboral, es allí donde se confirma o desvirtúa al egresado la calidad de educación que recibió en la Universidad; la producción intelectual, medida en: patentes, registros, artículos, ponencias y otros productos fruto de la investigación; la diseminación del conocimiento que hace la institución para mostrarla al contexto externo con una propuesta extensiva, como describe Souza et al. (2020): la internacionalización como aporte a la calidad educativa.

3) De la formación docente como categoría de motivación, se tiene que "los saberes de la formación docente inicial se encuentran tensionados por los cambios culturales, entre los que se encuentran, las condiciones de sociabilidad que colaboran en construir los artefactos culturales, tecnológicos, digitales y los medios conectivos" (De Marco, 2020). Bajo esta situación, la formación actual de los docentes en IES va unida como categoría al cambio tecnológico; hoy los 


\section{La motivación}

docente

espacios virtuales de aprendizaje vienen siendo un baluarte importante para llevar la formación a espacios que antes era imposible llegar, y todo por el desarrollo tecnológico.

4) De la responsabilidad del docente como categoría motivacional, indica Fernández (2020), que se le otorga al docente en su quehacer educativo una vocación por el otro, formando parte de la fenomenología de la responsabilidad el cuidado y la confianza; se sumarían otras categorías intrínsecas que hacen que el docente se integre a sus actividades laborales, aprendidas en las distintas instancias de socialización, anterior a la actividad docente.

5) Del factor extrínseco, señala Vargas (2012), el peso que tiene la cultura de la organización educativa en la determinación de los sistemas de incentivos, como salarios, bonificaciones, entre otras.

Después de haber configurado el sistema categorial, se utiliza la técnica Delphi, la cual es una "técnica esencial para obtener resultados de investigación válidos" (Veugelers et al., 2020), o también se define como un proceso donde, a través de unos expertos, se obtiene una valoración adecuada. La investigación contó con el apoyo de diez (10) expertos para evaluar el sistema categorial, con formación académica en docencia universitaria, los cuales a través de una primera ronda evaluarán cada una de las categorías; las ocho (8) con mayor reconocimiento quedaron para una segunda ronda; en la última ronda de expertos, seleccionaron las seis (6) con mayor valor, constituyendo así el sistema categorial que es tomado para hacer la descripción de tipo hermenéutico, "no hay hechos, sino sólo interpretaciones" (Nietzsche, como se citó en Grondin, 2019, p. 15). La investigación es de tipo descriptiva, porque busca dar cuenta de las características comportamentales de los docentes de las IES, en relación con el mejoramiento de la calidad educativa.

\section{Resultados}

La técnica Delphi, como lo indica Silva (2006), fue desarrollada en 1949 con fines de ser aplicada en estrategias militares; sin embargo, la técnica ha sido utilizada en infinidad de temáticas que la mantienen vigente. Para este caso, la investigación utilizó dos rondas de expertos quienes evalúan cada categoría, desde su conocimiento y experiencia, llegando a una mayor depuración del sistema categorial. En primera instancia, la clasificación de las categorías se hizo con 10 (diez) 


\section{La motivación}

docente

expertos, quienes evaluaron las catorce (14) categorías iniciales, llegando a seleccionar ocho (8) categorías, con un reconocimiento de hasta el $50 \%$, obteniendo los resultados que presenta la tabla 3.

\section{Tabla 3}

Técnica Delphi, primera ronda de expertos

\begin{tabular}{|c|c|c|c|c|c|c|c|c|c|c|}
\hline \multirow{2}{*}{ Categorías } & \multicolumn{10}{|c|}{ Expertos } \\
\hline & 1 & 2 & 3 & 4 & 5 & 6 & 7 & 8 & 9 & 10 \\
\hline 1. Acreditación de programa e institución & & $\mathrm{X}$ & & $\mathrm{X}$ & $\mathrm{X}$ & $\mathrm{X}$ & & & $\mathrm{X}$ & \\
\hline 2. Calidad en la educación & $X$ & $\mathrm{X}$ & & $\mathrm{X}$ & $\mathrm{X}$ & $\mathrm{X}$ & $\mathrm{X}$ & $X$ & $X$ & $X$ \\
\hline 3. Formación permanente & $\mathrm{X}$ & $\mathrm{X}$ & $\mathrm{X}$ & $\mathrm{X}$ & $\mathrm{X}$ & $\mathrm{X}$ & & $\mathrm{X}$ & $\mathrm{X}$ & $\mathrm{X}$ \\
\hline 4. Cuidado, confianza & & & & & & $\mathrm{X}$ & & $\mathrm{X}$ & $\mathrm{X}$ & \\
\hline 5. Responsabilidad en la enseñanza & $\mathrm{X}$ & & $\mathrm{X}$ & $\mathrm{X}$ & & & & & $\mathrm{X}$ & \\
\hline 6. Cambios tecnológicos & & & $\mathrm{X}$ & & $\mathrm{X}$ & & & $\mathrm{X}$ & & \\
\hline 7. Eficacia en la enseñanza & & & & & & & & & & \\
\hline 8. Factores intrínsecos (vocación) & $\mathrm{X}$ & $\mathrm{X}$ & $\mathrm{X}$ & $\mathrm{X}$ & $\mathrm{X}$ & $\mathrm{X}$ & $\mathrm{X}$ & $\mathrm{X}$ & $\mathrm{X}$ & $\mathrm{X}$ \\
\hline 9. Factores extrínsecos (salario, pensiones) & $\mathrm{X}$ & $\mathrm{X}$ & $\mathrm{X}$ & $\mathrm{X}$ & $\mathrm{X}$ & $\mathrm{X}$ & $\mathrm{X}$ & $\mathrm{X}$ & $\mathrm{X}$ & $\mathrm{X}$ \\
\hline 10. Rendimiento de los estudiantes & & $\mathrm{X}$ & & & $\mathrm{X}$ & & $\mathrm{X}$ & & & $\mathrm{X}$ \\
\hline 11. Entusiasmo para captar la atención & & & $\mathrm{X}$ & & & & $\mathrm{X}$ & & & \\
\hline $\begin{array}{l}\text { 12. La docencia como generadora de status } \\
\text { social }\end{array}$ & $\mathrm{X}$ & $\mathrm{X}$ & $X$ & & & & $\mathrm{X}$ & $X$ & & $\mathrm{X}$ \\
\hline $\begin{array}{l}\text { 13. Transformar del conocimiento, generar } \\
\text { investigación }\end{array}$ & $\mathrm{X}$ & $\mathrm{X}$ & $X$ & $\mathrm{X}$ & $X$ & $X$ & $X$ & $X$ & $X$ & $X$ \\
\hline 14. Libertad de cátedra & $\mathrm{X}$ & & & $\mathrm{X}$ & & $\mathrm{X}$ & $X$ & & & $X$ \\
\hline
\end{tabular}

Nota: elaboración propia.

En esta primera ronda, las categorías intrínsecas, extrínsecas y transformación del conocimiento reciben un $100 \%$ de aprobación, por parte de los expertos; la literatura corrobora el resultado, el cual ciñe el análisis de la motivación desde lo extrínseco e intrínseco, en Kizıltepe (2008), Franco, Vélez et al. (2018), Franco, López et al. (2020), Han \& Yin (2016). Por su parte, en la transformación del conocimiento, que es el eje articulador de la IES, con respecto de la investigación, coinciden los expertos con Cegarra (2012). Seguida está la calidad en la educación, que es un asunto latente en todo sistema educativo, y es el gran diferenciador entre unos y otros (Asencio et al., 2019; Orozco et al., 2020; R. Martínez et al., 2020).

Por su parte, como categoría en formación permanente, con un $90 \%$, como condición de mejoramiento y calidad en la educación, donde debe existir no solo el compromiso de formarse 


\section{La motivación}

docente

por parte de los docentes, sino, además, de las IES en formar al cuerpo profesoral (Asprelli, 2012; Bara, 2018). Continúa la docencia como generadora de estatus social, aspecto evidenciable más en unas sociedades que otras, enmarcadas por los valores y principios sociales, como en Finlandia, Corea del Sur, Canadá (Franco, 2013). Luego, la libertad de cátedra, con un $60 \%$; y, por último, la acreditación del programas e instituciones con un $50 \%$. La primera ronda permitió la eliminación de seis (6) categorías que obtuvieron valores inferiores al 50\%. Ahora, la tabla 4 muestra el resultado final hecha por los expertos en una segunda ronda.

\section{Tabla 4}

Resultados final primera ronda y categorías para la segunda ronda de expertos

\begin{tabular}{lc}
\hline \multicolumn{1}{c}{ Categoría seleccionada } & No. de respuestas indicadas \\
\hline 8. Factores intrínsecos (vocación) & 10 \\
\hline 9. Factores extrínsecos (salario, pensiones) & 10 \\
\hline 13 Transformar conocimiento, generar investigación & 10 \\
\hline 2. Calidad en la educación & 9 \\
\hline 3. Formación permanente & 9 \\
\hline 12 La docencia como generadora de estatus social & 6 \\
\hline 14 Libertad de cátedra & 6 \\
\hline 1. Acreditación del programa e institución & 5 \\
\hline
\end{tabular}

Nota: elaboración propia.

Pasada la primera ronda, donde fueron seleccionadas las ocho (8) categorías con el reconocimiento de hasta el $50 \%$, se configura nuevamente el sistema categorial y se entrega a diez (10) expertos para volver a efectuar una selección, con el ánimo de sacar el definitivo y último sistema categorial, dando como resultado lo que indica la tabla 5.

\section{Tabla 5}

Resultados final segunda ronda de expertos

\begin{tabular}{lrrrrrrrrrr}
\hline \multicolumn{1}{c}{ Categorías } & \multicolumn{11}{c}{ Expertos } \\
\cline { 2 - 11 } & $\mathbf{1}$ & $\mathbf{2}$ & $\mathbf{3}$ & $\mathbf{4}$ & $\mathbf{5}$ & $\mathbf{6}$ & $\mathbf{7}$ & $\mathbf{8}$ & $\mathbf{9}$ & $\mathbf{1 0}$ \\
\hline 1. 8. Factores intrínsecos (vocación) & $\mathrm{X}$ & $\mathrm{X}$ & & $\mathrm{X}$ & & $\mathrm{X}$ & $\mathrm{X}$ & $\mathrm{X}$ & $\mathrm{X}$ & $\mathrm{X}$ \\
\hline 2. 9. Factores extrínsecos (salario, pensiones) & $\mathrm{X}$ & $\mathrm{X}$ & $\mathrm{X}$ & $\mathrm{X}$ & $\mathrm{X}$ & $\mathrm{X}$ & $\mathrm{X}$ & $\mathrm{X}$ & $\mathrm{X}$ & $\mathrm{X}$ \\
\hline
\end{tabular}




\section{La motivación}

docente

3. 13 Transformar conocimiento, generar investigación

\begin{tabular}{|c|c|c|c|c|c|c|c|c|}
\hline 4. 2. Calidad en la educación & $\mathrm{X}$ & $\mathrm{X}$ & $\mathrm{X}$ & $\mathrm{X}$ & & $\mathrm{X}$ & $X$ & $\mathrm{X}$ \\
\hline 5. 3. Formación permanente & $\mathrm{X}$ & $\mathrm{X}$ & & & $\mathrm{X}$ & $\mathrm{X}$ & & $\mathrm{X}$ \\
\hline
\end{tabular}

6. 12 La docencia como generadora de status
social

\begin{tabular}{lllllll}
\hline 7. 14 Libertad de cátedra & $X$ & $X$ & $X$ & $X$ & & \\
\hline 8. Acreditación de programa e institución & $X$ & & $X$ & & $X$ & $X$ \\
\hline
\end{tabular}

Nota: elaboración propia.

La segunda ronda de 10 (diez) expertos produjo como resultado, con la técnica Delphi, la selección de seis (6) categorías, obteniendo los siguientes resultados: i) factores extrínsecos (salario, pensiones) con 10 respuestas favorables, y un $100 \%$ de reconocimiento; igual que en la primera ronda, su elección logró obtener el mayor nivel de reconocimiento por parte de los expertos. Es de anotar que en los docentes de IES arrojo este resultado, a diferencia de la anterior investigación en Franco, Vélez et al. (2018), en donde docentes de formación media, el mayor valor se concentró en otras categorías, como lo intrínseco. ii) Factores intrínsecos (vocación), con 8 respuestas indicadas, un $80 \%$ de favorabilidad, y con una disminución del $20 \%$, con respecto de la primera ronda; es menester indicar que estos factores intrínsecos, como la vocación, se insertan en la parte psíquica de cada persona y han sido desarrollados al interior de los distintos niveles de socialización que la persona haya tenido en su existencia. iii) Transformar conocimiento, con 7 respuestas favorables y un $70 \%$ de reconocimiento, disminuyo un $30 \%$, con respecto de la primera ronda; pese a lo anterior, entre la primera y segunda ronda la transformación y generación de conocimiento es la diferencia entre IES y, además, entre docentes que investigan y quienes no lo hacen. iv) Calidad en la educación, con 7 respuestas y un reconocimiento del 70 \%; disminuyó un $20 \%$ en comparación con la primera ronda. v) Formación permanente, con 5 respuestas, y un $50 \%$ de reconocimiento; disminuyó un $40 \%$ sobre la primera ronda. Y vi) La docencia como generadora de status social, con 5 respuestas y un $50 \%$ de reconocimiento; disminuye un $10 \%$ sobre la primera ronda. En la tabla 6 se indican los resultados finales, producto de los expertos en una segunda ronda, lo cual deja el sistema categorial para hacer un análisis final de tipo hermenéutico. 


\section{La motivación}

docente

\section{Tabla 6}

Resultados finales segunda ronda y categorías para el análisis hermenéutico

\begin{tabular}{lc}
\hline \multicolumn{1}{c}{ Categoría seleccionada } & No. de respuestas indicadas \\
\hline 8. Factores intrínsecos (vocación) & 10 \\
\hline 9. Factores extrínsecos (salario, pensiones) & 8 \\
\hline 13 Transformar conocimiento, generar investigación & 7 \\
\hline 2. Calidad en la educación & 7 \\
\hline 3. Formación permanente & 9 \\
\hline 12 La docencia como generadora de estatus social & 6 \\
\hline
\end{tabular}

Fuente: elaboración propia.

\section{Discusión}

De las categorías seleccionadas a través de la técnica Delphi se puede destacar en su orden: factores extrínsecos, como categoría en la motivación de los docentes de IES.

Esta se considera como el conjunto de recompensas monetarias, bien directas como pago de salarios, incentivos, complementos por méritos o indirectas como tiempo no trabajado, programas de protección, pagos en especie, y formación que a cambio de su trabajo, recibe un individuo. (Martín et al., 2009, p. 192)

Las actividades docentes en IES tienen niveles de exigencia, porque es allí donde se consolida finalmente el proceso de formación de los estudiantes en egresados, debe garantizar al educando las competencias para el mercado de trabajo o una posible formación adicional. Desde esa perspectiva, el docente tiene un gran reto en ser formador de conocimiento y en desarrollar actitudes; en esa perspectiva la remuneración salarial (factor extrínseco) debe ser retribuida como empleado del conocimiento, siendo este el principal recurso de las sociedades pos-capitalistas: "la última fase de la evolución del saber, cuando este se aplica al saber mismo, produce hoy una revolución de la gestión, pues el saber está deviniendo el factor número uno de la producción" (Druker, 1993, como se citó en Franco, Uribe et al., 2019, p. 162). Ahora, hay unos interrogantes de la motivación de los docentes de IES, con respecto de los factores extrínsecos como satisfactor de las necesidades básicas (como es el salario en este caso): ¿hasta qué tiempo durará el efecto motivacional en un docente IES como es el factor extrínseco salarial?; ¿bastará el factor extrínseco para mantener calidad en la educación? 


\section{La motivación}

docente

Ahora, el trabajo efectuado por Satizabal et al. (2020) describe la situación salarial de los docentes (como factor extrínseco); allí indica que las remuneraciones dependen del carácter de la institución (pública o privada) y la ubicación geográfica (urbana o rural), donde la baja remuneración y las amplias jornadas laborales (trabajo desde casa) es causal para abandonar la carrera docente. Asimismo, continúan los autores indicando que la incertidumbre, con respecto al futuro laboral, el empobrecimiento por la baja remuneración, y la flexibilidad del vínculo laboral, con niveles de contratación que no permiten estabilidad, son causales, entre otras, que hacen de la práctica docente una actividad poco llamativa para muchos.

De los factores intrínsecos, como categoría seleccionada, el estudio de López et al. (2017) concluyó que esta categoría en docentes de instituciones de educación secundaria, es la más decisiva; indican, también, que la vocación es lo que mueve a ejercer la profesión y hace que los docentes que la tengan, aseguren el éxito en el proceso de capacitación, y sean entregados a la tarea; "la vocación docente tiene un significativo de un componente afectivo, que ha estimulado a los docentes a desarrollar un compromiso social durante su labor educativa" (Mujica y Orellana, 2018). El efecto que generan los factores intrínsecos mantiene una duración por largo tiempo en el docente; lo anterior, genera la siguiente pregunta: en los docentes de IES, de igual manera que los docentes de educación secundaria, donde las condiciones del aprendizaje son diferentes por los actores del aprendizaje, ¿los factores intrínsecos tendrán la misma importancia?

Los factores intrínsecos mueven al docente desde su interior; eso que es difícil de precisar, porque está en la parte psíquica. Donovan (2002) indica que hay motivaciones o aspiraciones internas (intrínsecas) que mueven o llevan a ejecutar una tarea en espacios de presión e incertidumbre. "Los valores intrínsecos poseen una mayor influencia sobre el individuo que las gratificaciones externas como las recompensas, reconocimientos y, en general, incentivos externos

que comúnmente conocemos como estímulos" (Franco, López et al., 2020). En Mujica y Orellana (2018) se presenta un aspecto importante frente a la vocación docente, como categoría intrínseca, y es que bajo el escenario que mueve a los docentes a efectuar las actividades laborales, existe un abuso político-institucional que afecta la dignidad de los docentes, porque no hay una respuesta adecuada en términos salariales por parte del mercado privado o del Estado, es decir, los salarios recibidos por parte de los docentes, no compensan esa incondicionalidad de la vocación docente en el desarrollo de las actividades. 


\section{La motivación}

docente

De la transformación de conocimiento o generador de investigación; uno de los compromisos del cuerpo docente de las IES, es lograr que el cumplimiento del eje articulador de la investigación sea la desarrolladora de mejores condiciones de vida social, y se convierta en un compromiso con toda la comunidad académica. Del papel del docente de una IES, indica Cegarra (2012), que él tiene una doble función, ser docente e investigador; sin embargo, la realidad de las IES indica que el grado motivacional que tienen los docentes para abordar la investigación, como parte de sus actividades cotidianas, no es el común de todo el cuerpo docente, y se pregunta ¿qué motiva a un docente de una IES para ser un investigador?

De la calidad en la educación como categoría, se puede argumentar que es un eje transversal para aquellos docentes e instituciones que la adoptan como un estilo fundamental en la práctica del hacer cotidiano; la calidad en la educación es la garantía a una real transformación del contexto social. Hay una discusión que abre el trabajo de investigación de Vera y González (2018), cuando cuestionan la medición de la calidad en la educación, con indicadores establecidos por la política educativa gubernamental; aspectos como el número de académicos de tiempo completo con estudios de posgrado, número de estudiantes titulados, programas e instituciones acreditados, entre otros aspectos. Obviamente, lo anterior apunta a un mejoramiento; sin embargo, la calidad en la educación es una simbiosis de múltiples factores que parten de la voluntad del docente, de la institución y de las políticas gubernamentales, en términos de educación. "Aunque se evidencia una tradicional tendencia a poner el acento en resultados académicos medidos por pruebas estandarizadas, el concepto de calidad es polisémico y complejo” (Torche, Martínez, Madrid, 2015, como se citó en C. Rodríguez et al., 2020).

En relación con la formación permanente, indica Asprelli (2012) que enseñar es una actividad que se hace con la intención de dar lugar al aprendizaje, y es claro que la enseñanza requiere formación permanente por parte del docente; ahora, ¿en qué se debe formar para lograr enseñar? Es condición básica para generar y reproducir conocimiento y actitudes tener motivación al aprendizaje, el docente debe formarse en el discurso de la ciencia que irá a impartir, pero, además, debe saber aplicar la pedagogía y la didáctica. Docente que adolezca de tener una identidad al aprendizaje, estará condenado a repetir una y otra vez lo que alguna vez logró aprender. 


\section{La motivación}

docente

Y de la docencia como generadora de status social, hay que reconocerle la importancia que tiene en la actividad docente, en cualquier tipo de contexto social. Es innegable que el docente es un agente transformador de la sociedad; además, tiene un estatus reconocido, dependiendo del contexto situacional e histórico en el cual desarrolle su actividad. Sin embargo, el trabajo de Fernandes y Heloisa (2019) describe, en el contexto, los desafíos que viven los docentes en términos de sus condiciones de trabajo, estatus social y seguridad. De lo anterior, surge el interrogante: ¿hasta qué punto la profesión docente motiva, dependiendo del contexto situacional e histórico, a obtener estatus social?

De acuerdo con los hallazgos encontrados, se puede corroborar que las categorías seleccionadas por los 10 (diez) expertos con mayor reconocimiento, son los factores extrínsecos y factores intrínsecos, en cierta manera confirmados por la literatura; destacándose el trabajo de Rossi et al. (2020), cuando indican que la autoeficacia y la motivación intrínseca en el aprendizaje tienen una correlación positiva en los estudiantes de secundaria. De igual manera, Franco, Vélez et al. (2018) encontraron que los docentes de formación secundaria son motivados por factores intrínsecos, siendo la vocación el de mayor peso en el estudio; "la vocación es la característica o factor intrínseco más relevante, que impulsa a las personas a adoptar esta actividad como parte de su proyecto de vida, es el factor motivante que determinar (sic) la decisión de convertirse en docentes" (p. 165). Los factores intrínsecos motivan a los docentes y estudiantes de colegios. No obstante, en las IES son los factores extrínsecos los que motivan a los docentes; las retribuciones de orden monetario, bonificaciones, entre otras, contribuyen a mejores condiciones de vida, a mejorar el estatus y el prestigio social, logrando que esta categoría mueva al grupo de docentes.

\section{Conclusiones}

El docente en IES, como agente transformador del conocimiento, requiere tener capacidades y habilidades particulares que lo hacen creador y multiplicador, alrededor de las ideas expuestas frente a los estudiantes; ahora, ¿Qué lo mueve a desarrollar todo esto? Una de las respuestas es el grado motivacional que debe tener y la disponibilidad para enfrentar el día a día que le permita tener calidad en el proceso. La investigación llevó a reconocer, después de una búsqueda en expertos, que la motivación docente va dirigida alrededor de seis categorías o 


\section{La motivación}

docente

variables estratégicas: Factores extrínsecos, donde en ambas rondas de expertos obtuvo el mayor reconocimiento, y serían todos esos aspectos externos los que mejoran las condiciones de vida de los docentes; esos mayores ingresos que permiten escalonar la estructura social, y así tener, a la manera capitalista, un estatus, porque el ingreso le permite una diferenciación en el consumo; un hallazgo lleno de pragmatismo. Un segundo hallazgo de factores o categoría es lo intrínseco, lo que permite observar la visión sensible y llena de humanidad que tiene el docente en su práctica. La motivación parte desde el interior del ser, donde ubica la actividad docente; aquí el profesional se presenta en una visión altruista, porque lo mueve la vocación al servicio, ese deseo de dar sin condiciones, más allá del aprendizaje de los estudiantes y el mejoramiento institucional.

Otra motivación docente, en algunas IES, es la transformación del conocimiento, siendo la investigación un objetivo de gran alcance; solo la pueden adquirir los docentes que tienen como vocación una actividad compleja, pero gratificante. La transformación del conocimiento es posible para esos docentes que buscan verdaderas transformaciones; solo es posible convertirse en un docente investigador a través de la motivación al cambio, los que no buscan repetir semestre tras semestre el mismo discurso.

Es importante profundizar más en la propuesta; el tema de la motivación docente debe ahondar más, porque los causales históricas vienen cambiando en gran manera; el desarrollo tecnológico está haciendo cambiar la forma comportamental de los distintos agentes del aprendizaje; las aulas de clase presenciales ya vienen cambiando por espacios virtuales de aprendizaje; y el control de la información, que antes era exclusividad del docente, hoy es también de los estudiantes, en tanto pueden estar corroborando la información ofrecida en tiempo real, a través de las distintas plataformas tecnológicas. Estos y muchos aspectos vienen cambiando la educación en forma abrupta.

\section{Referencias}

Alexander, P. (2008). Charting the course for the teaching profession: The energizing and sustaining role of motivational forces. Learning and Instruction, 18(5), 483-491. https://dariasoi.org/10.1016/j.learninstruc.2008.06.006 


\section{La motivación}

docente

La motivación docente | Revista Virtual

Universidad Católica del Norte, 64, 151-179

ISSN: 0124-5821 (En línea)

Arias-Ciro, J. (2020). Estudio bibliométrico de la eficiencia del gasto público en educación. Revista CEA, 6(11), 127-144. https://doi.org/10.22430/24223182.1588

Asencio, A., Barrios, Y., Chuquihuara, A., De la Cruz, D., y Munares, O. (2019). Análisis crítico e histórico de la calidad de educación médica en el Perú. Eduación Médica Superior, 33(1), artículo e1413. http://www.ems.sld.cu/index.php/ems/article/view/1413

Asprelli, M. (2012). La didáctica en la formación docente. Homo Sapiens ediciones.

Atkinson, S. (2000). An investigation into the relationship between teacher motivation and pupil $\begin{array}{llll}\text { motivation. } & \text { Educational } & \text { Psychology, } & \text { 20(1), }\end{array}$ https://doi.org/10.1080/014434100110371

Bailey, J. (1999). Academics' Motivation and Self-efficacy for Teaching and Research. Higher Education Research \& Development, 18(3), 343-359. https://doi.org/10.1080/0729436990180305

Baleghizadeh, S., \& Gordani, Y. (2012). Motivation and quality of work life among secondary shool EFL teachers. Australian Journal of Teacher Education, 37(3), 30-42. https://doi.org/10.14221/ ajte.2012v37n7.8

Bara, F. (2018). Ética del profesor. Herder Editorial. https://elibronet.itm.elogim.com:2443/es/ereader/bibliotecaitm/116586?as_all=Profesor\&as_all_op=u naccent_icontains \&prev=as

Brookhart, S., y Freeman, D. (1992). Características de los candidatos a maestros entrantes. Research in Education, 62(1), 37-60. https://doi.org/10.3102/00346543062001037

Cegarra, J. (2012). El investigador y sus características. Díaz de Santos. https://elibronet.itm.elogim.com:2443/es/ereader/bibliotecaitm/62636?fs_q=\%C2\%BFqu\%C3\%A9 es investigar\&prev $=\mathrm{fs}$

Consejo Nacional de Educación Superior -CESU-. (1 de julio de 2020). Acuerdo 02, por el cual se actualiza el modelo de acreditación en alta calidad. https://www.mineducacion.gov.co/1759/articles-399567_recurso_1.pdf

De Jesus, S., \& De Willy, L. (2004). An integrated model for the study of teacher motivation. Applied Psychology, 54(1), 119-134. https://doi.org/10.1111/j.1464-0597.2005.00199.x 


\section{La motivación}

docente

La motivación docente | Revista Virtual

Universidad Católica del Norte, 64, 151-179

ISSN: 0124-5821 (En línea)

De Marco, L. (2020). Formación docente y cambios culturales contemporáneos. Nuevos saberes pedagógicos para otras condiciones de sociabilidad. Espacios en Blanco. Serie Indagaciones, 30(2), 293-306. https://doi.org/10.37177/UNICEN/EB30-278

Deci, E., \& Ryan, R. (2008). Self-Determination Theory: A Macrotheory of Human Motivation, develotment, and health. Canadian Psychology, 49(3), 182-185. https://doi.org/10.1037/a0012801

Donovan, J. (2002). Work motivation. In N. Anderson, D. S. Ones, H. K. Sinangil, \& C. Viswesvaran (Eds.), Handbook of industrial, work and organizational psychology (Vol. 2, pp. 53-76). Sage Publications, Inc. https://psycnet.apa.org/record/2003-00438-003

Dörnyei, Z., y Ushioda, E. (2012). Enseñar a investigar la motivación (Vol. 2). Person Education Limited.

Fattash, M. (2013). Demotivating Factors of University ESL Teachers. International Journal of

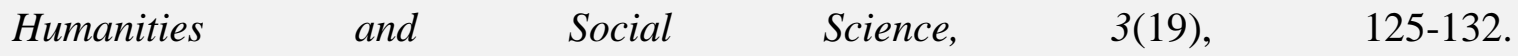
http://www.ijhssnet.com/journals/Vol_3_No_19_November_2013/15.pdf

Fernandes, S., y Heloisa, R. (2019). A Estrutura das Representações Sociais acerca del Professor entre os Pais de Alunos. Tendencias en Psicología, 27(1), 265-278. https://doi.org/doi.org/10.9788/tp2019.1-19

Fernández, G. (2020). Fenomenología de la responsabilidad por el Otro: un estudio sobre la vocación de la docencia. Educación, 44(1). https://doi.org/10.15517/revedu.v44i1.39145

Fong, C., Dillard, J., \& Hatcher, M. (2019). Teaching self-efficacy of graduate student instructors: Exploring faculty motivation, perceptions of autonomy support, and undergraduate student engagement. Journal of Educational Research, 98, 91-105. https://doi.org/10.1016/j.ijer.2019.08.018

Fox, R. (1961). Factors influencing the career choice of prospective teachers. Journal of Teacher Education, 12, 427-432. https://doi.org/10.1177/002248716101200410

Franco, J. (2013). Experiencias exitosas en educación. La Tekhné, (74), 2. https://www.itm.edu.co/wpcontent/uploads/2016/05/PDF_LA_TEKHNE_74_Agosto_de_2013.pdf 


\section{La motivación}

docente

La motivación docente | Revista Virtual

Universidad Católica del Norte, 64, 151-179

ISSN: 0124-5821 (En línea)

Franco, J., López, H., y Arango, D. (2020). La satisfacción se ser docente: un estudio de tipo correlacional. Complutense de Educación, 31(1), 55-67. https://doi.org/10.5209/rced.61775

Franco, J., Uribe, A., y Monsalve, J. (2019). El capital humano y estructural a través de lógica difusa 2. Lasallista de Investigación, 16(2), 160-170. https://doi.org/10.22507/rli.v16n2a13

Franco, J., Vélez, F., y López, H. (2018). La motivación docente y su repercusión en la calidad eduactiva: estudio de caso. Revista de Pedagogía, 39(105), 151-172. http://saber.ucv.ve/ojs/index.php/rev_ped/article/view/16530

Fuchs, C. (2018). Industry 4.0: The Digital German Ideology. TripleC, 16(1), 280-289. https://doi.org/10.31269/vol16iss1pp280-289

Galeano, E. (2004). Diseño de proyectos de investigación cualitativa. Fondo Editorial Universidad Eafit.

https://books.google.com.co/books?id=Xkb78OSRMI8C\&printsec=\%20frontcover\&hl=e

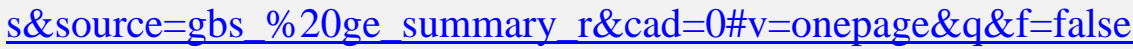

Ganga, F., Reyes, J., Rojas, C., Estay, J., Cabezas, C., y Vargas, S. (2018). Educación y Calidad: Análisis comparado del desempeño estudiantil en contextos vulnerables en una provincia de

Chile.

Espacios,

$39(52)$. http://www.revistaespacios.com/a18v39n52/a18v39n52p36.pdf

Gong, Z., Chen, Y., \& Wang, Y. (2019). The Influence of Emotional Intelligence on Job Burnout and Job Performance: Mediating Effect of Psychological Capital. Frontiers in Psychology, 10, 1-11. https://doi.org/10.3389/fpsyg.2019.02707

Grondin, J. (2019). ¿Qué es hermenéutica? Herder Editorial. https://elibronet.itm.elogim.com:2443/es/lc/bibliotecaitm/titulos/45769?prev=as

Guerrero, G., y Guerrero, M. (2014). Metodología de la investigación. Grupo Editorial Patria. https://elibro-

net.itm.elogim.com:2443/es/ereader/bibliotecaitm/40363?as_all=Investigaci\%C3\%B3n\% 20descriptiva\&as_all_op=unaccent_icontains\&prev=as

Han, J., \& Yin, H. (2016). Teacher motivation: Definition, research development and implications for teachers. Cogent Education, 3(1). https://doi.org/10.1080/2331186X.2016.1217819 


\section{La motivación}

docente

La motivación docente | Revista Virtual

Universidad Católica del Norte, 64, 151-179

ISSN: 0124-5821 (En línea)

Horodnic, I., \& Zait, A. (2015). Motivation and research productivity in a university system undergoing transition. Research evaluation, 24(3), 282-292. https://doi.org/10.1093/reseval/rvv010

Hotaman, D., \& Yüksel, F. (2010). The Effect of Instructors' Enthusiasm on University Students' Level of achievement motivation. Education and Science, 35(155), 89-103. https://pdfs.semanticscholar.org/4338/e16192ecc889c6d1ea354f3c7f0d489a3324.pdf

Ibrahim, A., \& Zaatari, W. (2020). The teacher-student relationship and adolescents' sense of school belonging. International Journal of Adolescence and Youth, 251, 382-395. https://doi.org/10.1080/02673843.2019.1660998

Ibrahim, M., \& Nat, M. (2019). Blended learning motivation model for instructors in higher education institutions. International Journal of Educational Technology in Higher, 16(12). https://doi.org/10.1186/s41239-019-0145-2

Kiziltepe, Z. (2008). Motivation and demotivation of university teachers. Teachers and Teaching, 14, 515-530. https://doi.org/10.1080/13540600802571361

Loaiza-Aguirre, M. I., y Andrade-Abarca, P. S. (mayo-agosto, 2021). Análisis de programas de desarrollo profesional del profesorado universitario. Revista Virtual Universidad Católica del Norte, (63), 161-195. https://www.doi.org/10.35575/rvucn.n63a7

López, H., Vélez, M., y Franco , J. (2017). Percepciones acerca de la motivación docente en personal directivo de institucioners de educación secundaria en la zona metropolitana de Medellín, 2015. Educare, 21(2), 1-23. https://doi.org/10.15359/ree.21-2.5

Martín, N., Trevilla, C., y Martín, V. (2009). Influencia de la motivación intrínseca y extrínseca sobre la transmisión de conocimiento. El caso de una organización sin animo de lucro. Revista de Economía Pública, Social y Cooperativa, (66), 187-211. https://www.redalyc.org/articulo.oa?id=17413043009

Martínez, C., Hervás, C., y Román, P. (2017). Experiencia en el ámbito universitario: autoeficacia y motivación docente. International Journal of Educational Reservarch and Innovation, 8 , 175-184. https://www.upo.es/revistas/index.php/IJERI/article/view/2403

Martínez, R., Sánchez, I., Gónzalez, D., \& Llamas, J. (2020). Strategies for attention to diversity: Perceptions of secondary school teaching staff. International Journal of Environmental Research and Public Health, 17(11). https://doi.org/10.3390/ijerph17113840 


\section{La motivación}

docente

La motivación docente | Revista Virtual

Universidad Católica del Norte, 64, 151-179

ISSN: 0124-5821 (En línea)

Ministerio de Educación Nacional. (2016). Compendio estadístico de la eduación superior en Colombia.

Imprenta

Nacional

de

Colombia. https://www.mineducacion.gov.co/1759/articles-360739_recurso.pdf

Mujica, F., y Orellana, N. (2018). Autopercepción de la vocación en docentes de educación física escolar en Chile. Investigación Educativa, (27), 203-229. https://doi.org/10.25009/cpue.v0i27.2563

Nie, Y., Lau, S., \& Liau, A. (2012). The Teacher Efficacy Scale: A realiability and validity study. The Asia-Pacific Education Researcher, (21), 414-421. https://repository.nie.edu.sg/bitstream/10497/14287/1/TAPER-21-2-414.pdf

Orozco, E., Jaya, A., Ramos, F., y Guerra, R. (2020). Restos de la gestión de la calidad en las instituciones de educación superior en Ecuador. Educación Médica Superior, 34(2), artículo e2268. http://www.ems.sld.cu/index.php/ems/article/view/2268

Pan, Y., \& Hu, C. (2020). Alleviation of job burnout of teachers through construction of psychological capital. Argentina de Clínica Psicológica, 29(1), 1216-1221. https://dialnet.unirioja.es/servlet/articulo?codigo $=7807193$

Penprase, B. (2018). The fourth industrial revolution and higher education. In N. Gleason (Ed.), Higher educatin in the era of the fourth industrial revolution (pp. 207-229). Springer. https://link.springer.com/chapter/10.1007\%2F978-981-13-0194-0_9

Pola, Á. (1988). Gestión de la calidad. Marcombo. https://elibronet.itm.elogim.com:2443/es/ereader/bibliotecaitm/45847?as_all=concepto\%20calidad\&a s_all_op=unaccent_icontains\&prev=as

Praver, M., \& Oga-Baldwin, W. (2008). What motivates language teachers: investigating work satisfaction and second language pedagogy. Polyglossia, 14, 1-8. https://en.apu.ac.jp/rcaps/uploads/fckeditor/publications/polyglossia/Polyglossia_V14_M ax_William.pdf

Richardson, P., \& Watt, H. (2006). Who chooses teaching and why? Profiling characteristics and motivations across Australian Universities. Asia-Pacific Journal of Teacher Education, 34(1), 27-56. https://doi.org/10.1080/13598660500480290

Rodríguez, C., Padilla, G., y Gallegos, M. (2020). Calidad educativa, apoyo docente y familiar percibido: la tridimensionalidad de la satisfacción escolar en niños y adolescentes. 


\section{La motivación}

docente
La motivación docente | Revista Virtual Universidad Católica del Norte, 64, 151-179 ISSN: 0124-5821 (En línea)

Cuadernos de Investigación Educativa, 11(2), 157-173. https://doi.org/10.18861/cied.2020.11.2.2995

Rodríguez, E., Schneider, D., \& Keenan, E. (2013). Being Known in Undergraduate Social Work Education: The Role of Instructors in Fostering Student Engagement and Motivation. Social Work Education, 326, 785-799. https://doi.org/10.1080/02615479.2013.765841

Rossi, T., Trevisol, A., Santos, D., Dapieve, N., y Hohendorff, J. (2020). Autoeficacia general percibida y motivación para aprender en adolescentes de secundaria. Acta Colombiana de Psicología, 23(1), 264-271. https://doi.org/10.14718/acp.2020.23.1.12

Satizabal, M., Cruz, A., y Unás, V. (2020). Condiciones de empleo de un grupo de docentes en Cali, Colombia. Entramado, 16(1), 108-120. https://doi.org/10.18041/1900$\underline{3803 / \text { entramado.1.6080 }}$

Serrate, S., Casillas, S., y Cabezas, M. (2019). Factores de calidad determinantes de la formación práctica de los estudiantes de educación. Ensaio: Avaliação e Políticas Públicas em Educação, 27(105), 817-838. https://doi.org/10.1590/s0104-40362019002701625

Silva, A. (2006). Nivel de satisfacción del ususrio de las carreteras: método Delphi. Red Acta Universitaria.

Sinclair, C. (2008). Initial and changing student teacher motivation and commitment to teaching. Asia-Pacific. Journal of Teacher Education, 36(2), 79-104. http://dx.doi.org/10.1080/13598660801971658

SNIES. (2020). Información poblacional. https://hecaa.mineducacion.gov.co/consultaspublicas/poblacionalies?p_anio=2016\&p_se $\underline{\text { mestre }}=2 \& p \_$variable $=$DOCENTE $\& p \_$depto $=C O L$

Souza, C., Filippo, D., y Casado, E. (2020). El papel de la internacionalización de la educación superior en la producción científica brasileña. Ensaio: Avaliação e Políticas Públicas em Educação, 28(108), 784-810. https://doi.org/10.1590/s0104-40362019002701721

Sugino, T. (2010). Teacher demotivational factors in the Japanese language teaching context. Procedia: scoail and Behavioral, 3, 216-226. https://doi.org/10.1016/j.sbspro.2010.07.036

Tae, K., Yoon, K., \& Quian, Z. (2014). Differences in Demotivation Between Chinese and Korean English Teachers: A mixed-methods study. The Asia-Pacific Education Researcher, 23(2), 299-310. https://doi.org/10.1007/s40299-013-0105-x 


\section{La motivación}

docente

Thoonen, E., Sleegers, P., Oort, F., Peetsma, T., \& Geijsel, F. (2011). How to Improve Teaching Practices: The Role of Teacher Motivation, Organizational Factors, and Leadership Practices. Educational Administration Quarterly, 47(3), 496-536. https://doi.org/10.1177/0013161X11400185

Vargas, J. (2012). Implicaciones de la teoría motivacional de la Autodeterminación en el ámbito

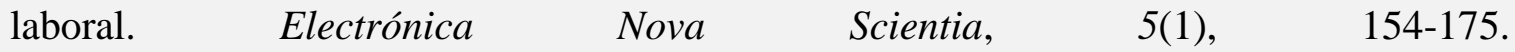
http://www.scielo.org.mx/pdf/ns/v5n9/v5n9a10.pdf

Vera, H., y González, M. (2018). Calidad y evaluación: matrimonio y el infierno. Perfiles Educativos, 40(especial), 53-97. https://doi.org/10.22201/iisue.24486167e.2018.Especial.59180

Veugelers, R., Gaakeer, M., Patka, P., \& Huijsman, R. (2020). Improving design choices in Delphi studies in medicine: The case of an exemplary physician multi-round panel study with 100\% response. BMC Medical Research Methodology, 20(156). https://doi.org/10.1186/s12874-020-01029-4 